\title{
Analysis of Complications of Percutaneous Transthoracic Needle Biopsy Using CT-Guidance Modalities In a Multicenter Cohort of 10568 Biopsies
}

\author{
Soon Ho Yoon, $M D^{1,2}$, Chang Min Park, MD, PhD ${ }^{1,2}$, Kyung Hee Lee, MD ${ }^{3}$, Kun Young Lim, MD ${ }^{4}$, \\ Young Joo Suh, $M D^{5}$, Dong Jin Im, $M D^{5}$, Jin Hur, $M D^{5,6}$, Dae Hee Han, MD${ }^{7}$, Mi-Jin Kang, $\mathrm{MD}^{8}$, \\ Ji Yung Choo, $M D^{9}$, Cherry Kim, $M D^{9}$, Jung Im Kim, MD ${ }^{10}$, Hyunsook Hong, PhD ${ }^{11}$
}

\begin{abstract}
${ }^{1}$ Department of Radiology, Seoul National University College of Medicine, Seoul, Korea; ${ }^{2}$ Institute of Radiation Medicine, Seoul National University Medical Research Center, Seoul, Korea; ${ }^{3}$ Department of Radiology, Seoul National University Bundang Hospital, Seongnam, Korea; ${ }^{4}$ Department of Radiology, National Cancer Center, Goyang, Korea; ${ }^{5}$ Department of Radiology, Severance Hospital, Yonsei University College of Medicine, Seoul, Korea; ${ }^{6}$ Research Institute of Radiological Science, Severance Hospital, Yonsei University College of Medicine, Seoul, Korea; ${ }^{7}$ Department of Radiology, Seoul St. Mary's Hospital, College of Medicine, The Catholic University of Korea, Seoul, Korea; ${ }^{8}$ Department of Radiology, Inje University Sanggye Paik Hospital, Seoul, Korea; ${ }^{9}$ Department of Radiology, Korea University Ansan Hospital, Korea University College of Medicine, Ansan, Korea; ${ }^{10}$ Department of Radiology, Kyung Hee University Hospital at Gangdong, College of Medicine, Kyung Hee University, Seoul, Korea; ${ }^{11}$ Medical Research Collaborating Center, Seoul National University Hospital, Seoul, Korea
\end{abstract}

Objective: To analyze the complications of percutaneous transthoracic needle biopsy using CT-based imaging modalities for needle guidance in comparison with fluoroscopy in a large retrospective cohort.

Materials and Methods: This study was approved by multiple Institutional Review Boards and the requirement for informed consent was waived. We retrospectively included 10568 biopsies from eight referral hospitals from 2010 through 2014. In univariate and multivariate logistic analyses, 3 CT-based guidance modalities (CT, CT fluoroscopy, and cone-beam CT) were compared with fluoroscopy in terms of the risk of pneumothorax, pneumothorax requiring chest tube insertion, and hemoptysis, with adjustment for other risk factors.

Results: Pneumothorax occurred in 2298 of the 10568 biopsies (21.7\%). Tube insertion was required after 316 biopsies $(3.0 \%)$, and hemoptysis occurred in 550 cases $(5.2 \%)$. In the multivariate analysis, pneumothorax was more frequently detected with CT \{odds ratio (OR), 2.752 (95\% confidence interval $[C I], 2.325-3.258), p<0.001$ \}, CT fluoroscopy (OR, 1.440 [95\% CI, 1.176-1.762], $p<0.001$ ), and cone-beam CT (OR, 2.906 [95\% CI, 2.235-3.779], $p<0.001)$, but no significant relationship was found for pneumothorax requiring chest tube insertion $(p=0.497, p=0.222$, and $p=0.216$, respectively). The incidence of hemoptysis was significantly lower under CT (OR, 0.348 [95\% CI, 0.247-0.491], $p<0.001)$, CT fluoroscopy $(0 R, 0.594$ [95\% CI, 0.419-0.843], $p=0.004)$, and cone-beam CT (OR, 0.479 [95\% CI, 0.317-0.724], $p<0.001)$ guidance. Conclusion: Hemoptysis occurred less frequently with CT-based guidance modalities in comparison with fluoroscopy. Although pneumothorax requiring chest tube insertion showed a similar incidence, pneumothorax was more frequently detected using CT-based guidance modalities.

Keywords: Lung neoplasms; Biopsy; Image-guided biopsy; Pneumothorax; Hemoptysis; CT; CT fluoroscopy; Cone-beam CT

Received January 24, 2018; accepted after revision August 6, 2018.

This study was supported by a grant of the Korea Health Technology R\&D Project through the Korea Health Industry Development Institute (KHIDI), funded by the Ministry of Health \& Welfare, Republic of Korea (grant number: HI15C1234).

Corresponding author: Chang Min Park, MD, PhD, Department of Radiology, Seoul National University College of Medicine, Institute of Radiation Medicine, Seoul National University Medical Research Center, 101 Daehak-ro, Jongno-gu, Seoul 03080, Korea.

- Tel: (822) 2072-0367•Fax: (822) 743-6386•E-mail: cmpark.morphius@gmail.com

This is an Open Access article distributed under the terms of the Creative Commons Attribution Non-Commercial License (https:// creativecommons.org/licenses/by-nc/4.0) which permits unrestricted non-commercial use, distribution, and reproduction in any medium, provided the original work is properly cited. 


\section{INTRODUCTION}

Image-guided percutaneous transthoracic needle biopsy (PTNB) plays a key role in the pathologic evaluation of pulmonary parenchymal lesions $(1,2)$. Fluoroscopy was the conventional guidance modality of choice for PTNB, but with the development of $\mathrm{CT}$ technologies, CT-based guidance modalities such as CT, CT fluoroscopy, and cone-beam CT have been introduced for PTNB. These CT-based modalities allow more accurate needle placement than that achieved with fluoroscopy, and the diagnostic accuracy of PTNB conducted using these modalities is as high as $97 \%(3,4)$.

Although PTNB has been recognized as a safe and relatively noninvasive diagnostic procedure, it is inevitably accompanied by a risk of procedure-related complications (5). In a recent meta-analysis of the complication rates of CT-guided PTNB (6), minor complications of core biopsy and fine-needle aspiration (FNA) were commonly observed with rates of $38.8 \%$ (95\% confidence interval [CI], 34.3$43.5 \%$ ) and $24.0 \%$ (95\% CI, 18.2-30.8\%), whereas major complications were infrequent, with rates of $5.7 \%(95 \% \mathrm{CI}$, 4.4-7.4\%) and 4.4\% (95\% CI, 2.7-7.0\%), respectively. In comparison with fluoroscopic guidance, CT-based guidance modalities may prove beneficial for avoiding procedurerelated complications by facilitating precise planning and secure placement of the biopsy needle (4). However, the impact of these CT-based imaging modalities on the complications of PTNB has not yet been critically evaluated in comparison with conventional fluoroscopic guidance, and there is limited comparison of the complication rates in small numbers of patients across CT-based guidance modalities (7-9).

Thus, we sought to analyze the complications of PTNB performed using CT-based imaging modalities for needle guidance in comparison with that performed using fluoroscopy in a large retrospective cohort.

\section{MATERIALS AND METHODS}

\section{Study Design and Population}

The Korean Registry of Percutaneous Needle Lung Biopsy group was organized to perform this multi-institutional cohort study. We retrospectively collected information about consecutive patients who underwent PTNBs at eight participating hospitals from January 2010 through December 2014. The participating institutions consisted

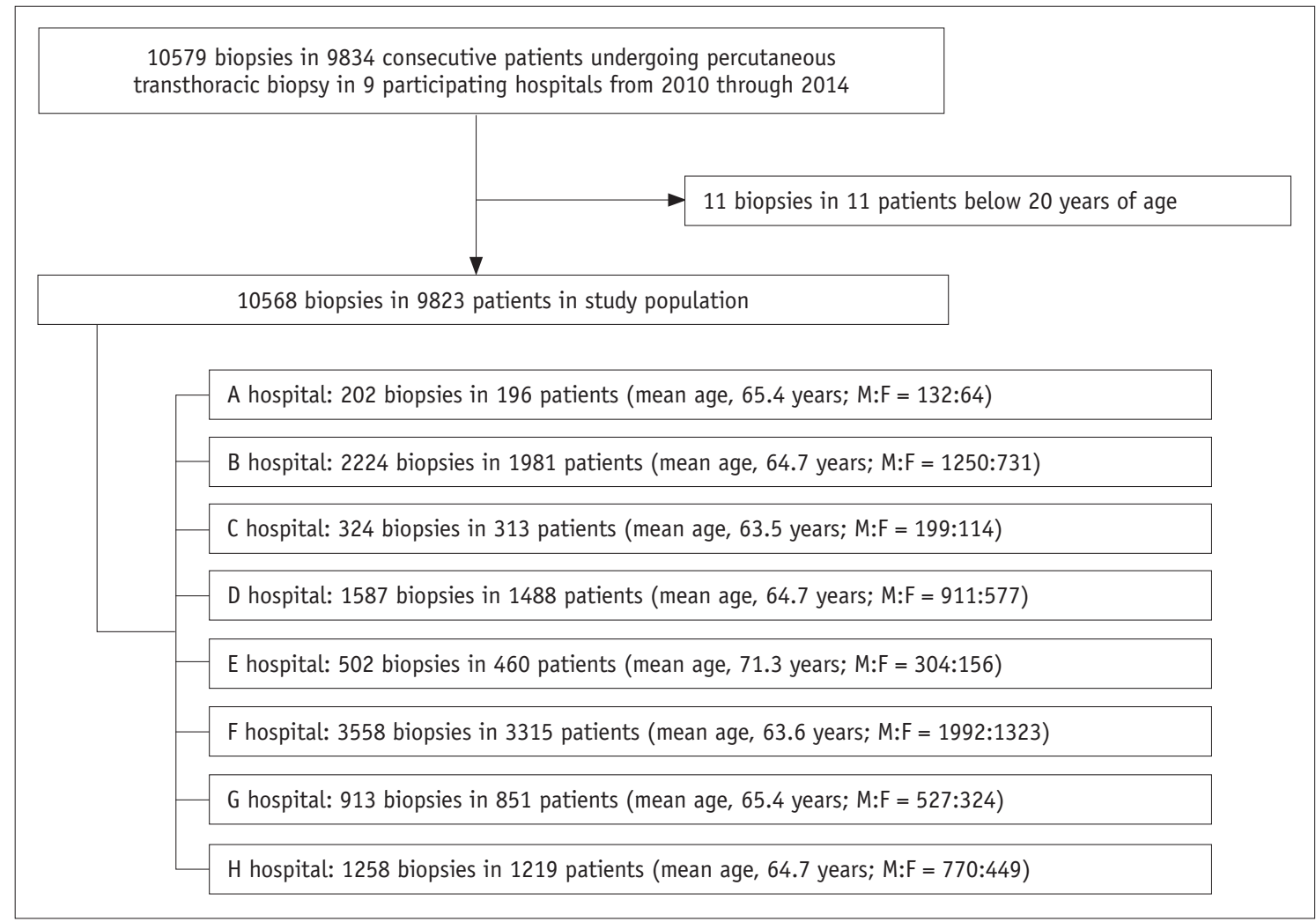

Fig. 1. Flow diagram of patient inclusion. $F=$ female, $M=$ male 
of six tertiary and two secondary referral hospitals in the metropolitan area of Seoul, with 555-2320 hospital beds.

We included patients aged at least 20 years who had undergone PTNB and had available preprocedural chest CT images. During the study period, a total of 10568 biopsies in 9823 patients (mean age, $64.7 \pm 11.8$ years; male-tofemale ratio, 6085:3738) were included in this study (Fig. 1). PTNBs were performed twice in 639 patients and 3 times or more in 44 patients: 502 biopsies for the same lesion within one year of an initial biopsy; 151 biopsies for the same lesion one year after the initial biopsy; and 85 biopsies for a different lesion. Repeat biopsies for the same lesion and biopsies for different lesions were considered to be separate PTNBs. The number of patients included from the participating institutions ranged from 196 to 3315 patients (median, 1035 patients). This study was approved by the Institutional Review Board of each participating hospital, and the requirement for informed consent from patients was waived.

\section{Biopsy Procedures}

In each participating hospital, PTNB was performed primarily by a dedicated thoracic radiologist who had performed at least 100 PTNBs before 2010, but training residents or fellows were sometimes involved in a particular portion of the PTNB procedure under the supervision of the thoracic radiologist. The participating institutions used different principal guidance modalities with a coaxial or non-coaxial needle (Table 1).

\section{Data Collection}

Data collection commenced in April 2016 and the data were locked as of August 2017. A trained nurse or designated investigator at the participating institutions collected information regarding patient and lesion characteristics using a standardized form, along with detailed information about the biopsy procedure. The patient characteristics included age, sex, smoking history, and emphysema, which was visually assessed on preprocedural CT images by a designated investigator. The lesion characteristics encompassed the long-axis diameter of the lesion, internal composition (solid versus subsolid), lobar location, and the distance from the pleura to the target lesion. Information about the biopsy procedure consisted of the guidance modality, biopsy method (core biopsy versus $F N A$ ), use of a coaxial needle, biopsy needle size $(22 \mathrm{G}, 20 \mathrm{G} / 19 \mathrm{G}$, or $18 \mathrm{G} / 17 \mathrm{G})$, number of tissue samples, and number of pleural passages. If a coaxial needle was used, the size of the outer coaxial needle was recorded.

Complications, including pneumothorax, pneumothorax requiring chest tube insertion, hemoptysis, and other rare complications, were assessed by reviewing the medical records that were created during the procedure and up to a week after. In fluoroscopy- or US-guided PTNBs, the presence of PTNB-related pneumothorax was assessed on the basis of chest radiographs, which were typically taken 3 hours after the PTNB. Intraprocedural and post-procedural CT images were used to determine the presence of PTNBrelated pneumothorax in CT-guided, CT fluoroscopy-guided, or cone-beam CT-guided PTNBs.

\section{Statistical Analysis}

The guidance modalities and usage of a coaxial needle were extremely biased according to the participating hospital (Table 1). Accordingly, hospital-associated effects were not appropriately separated from the effects of the guidance modalities and usage of a coaxial needle. As all

Table 1. Guidance Modalities and Usage of Coaxial Needle for Percutaneous Transthoracic Needle Biopsy According to Participating Institutions

\begin{tabular}{|c|c|c|c|c|c|c|c|c|}
\hline & \multicolumn{8}{|c|}{ Participating Institutions } \\
\hline & A & B & C & D & $E$ & $\mathrm{~F}$ & G & $\mathrm{H}$ \\
\hline \multicolumn{9}{|l|}{ Guidance modalities (\%) } \\
\hline Fluoroscopy & 0 & 100 & 5 & 3 & 93 & 0 & 0 & 19 \\
\hline CT & 0 & 0 & 86 & 97 & 6 & 0 & 100 & 0 \\
\hline CT fluoroscopy & 0 & 0 & 0 & 0 & 0 & 0 & 0 & 76 \\
\hline Cone-beam CT & 97 & 0 & 0 & 0 & 0 & 100 & 0 & 0 \\
\hline Ultrasonography & 3 & 0 & 9 & 0 & 1 & 0 & 0 & 5 \\
\hline \multicolumn{9}{|c|}{ Usage of coaxial needle (\%) } \\
\hline Coaxial needle & 0 & 100 & 0 & 94 & 100 & 3 & 2 & 99 \\
\hline Non-coaxial needle & 100 & 0 & 100 & 6 & 0 & 97 & 98 & 1 \\
\hline
\end{tabular}


participating hospitals were major regional referral hospitals and the affiliated thoracic radiologists had similar levels of experience performing PTNBs, we acknowledged the limitations of our data and cautiously decided to analyze the impact of the guidance modalities on the complication rates of PTNB, while excluding a hospital effect. Univariate and multivariate logistic regression analyses were performed to identify risk factors for pneumothorax, pneumothorax requiring chest tube insertion, and hemoptysis. In the multivariate logistic regression analysis, all variables with a $p$ value of 0.10 or less were entered into the final model. Biopsy method was also included in the multivariate model though it had a $p$ value $>0.1$ in the univariate analysis, since the biopsy method was a main factor affecting the PTNB complication rate (6). $P$ values of 0.05 or smaller were considered to indicate statistical significance. Statistical analysis was performed using SPSS version 22.0 (IBM Corp., Armonk, NY, USA).

\section{RESULTS}

Patient and lesion characteristics are summarized in Table 2. Of the 10568 biopsies, pneumothorax occurred in $21.7 \%$ (95\% CI, 20.9-22.5\%; 2298 biopsies), with tube insertion subsequently required in 3.0\% (95\% CI, 2.7-3.3\%; 316 biopsies), while hemoptysis occurred in $5.2 \%$ (95\% CI, 4.8-5.6\%; 550 biopsies). Other rare complications included hemothorax in $0.57 \%$ of the biopsies (95\% CI, $0.43-0.73 \%$; 59 biopsies), vasovagal syncope in $0.07 \%$ ( $95 \% \mathrm{CI}$, $0.03-0.15 \% ; 7$ biopsies), intra-abdominal injury in $0.03 \%$ (95\% CI, 0.01-0.09\%; 3 biopsies), air embolism in $0.02 \%$ (95\% CI, $0.00-0.08 \% ; 2$ biopsies), and death in $0.01 \%$ ( $95 \%$ CI, $0.00-0.06 \%$; 1 biopsy). Among the 59 biopsies with hemothorax, $71.9 \%$ and $93.0 \%$ were performed in the prone position and with core biopsy, respectively, compared to biopsies without hemothorax $(60.0 \%$ and $54.4 \%$, respectively), while other characteristics were similar.

\section{Pneumothorax}

Univariate logistic regression analysis identified the following significant risk factors (Supplementary Table 1 in the online-only Data Supplement): older age $(p=0.001)$, male sex $(p<0.001)$, smoking history $(p<0.001)$, guidance modality $(p<0.001-0.003)$, lobar location $(p<0.001-$ $0.854)$, lesion diameter $(p<0.001)$, lesion composition ( $p$ $=0.002)$, emphysema $(p<0.001)$, a deep-seated lesion $(p$ $<0.001)$, biopsy method $(p<0.001)$, needle gauge $(p<$

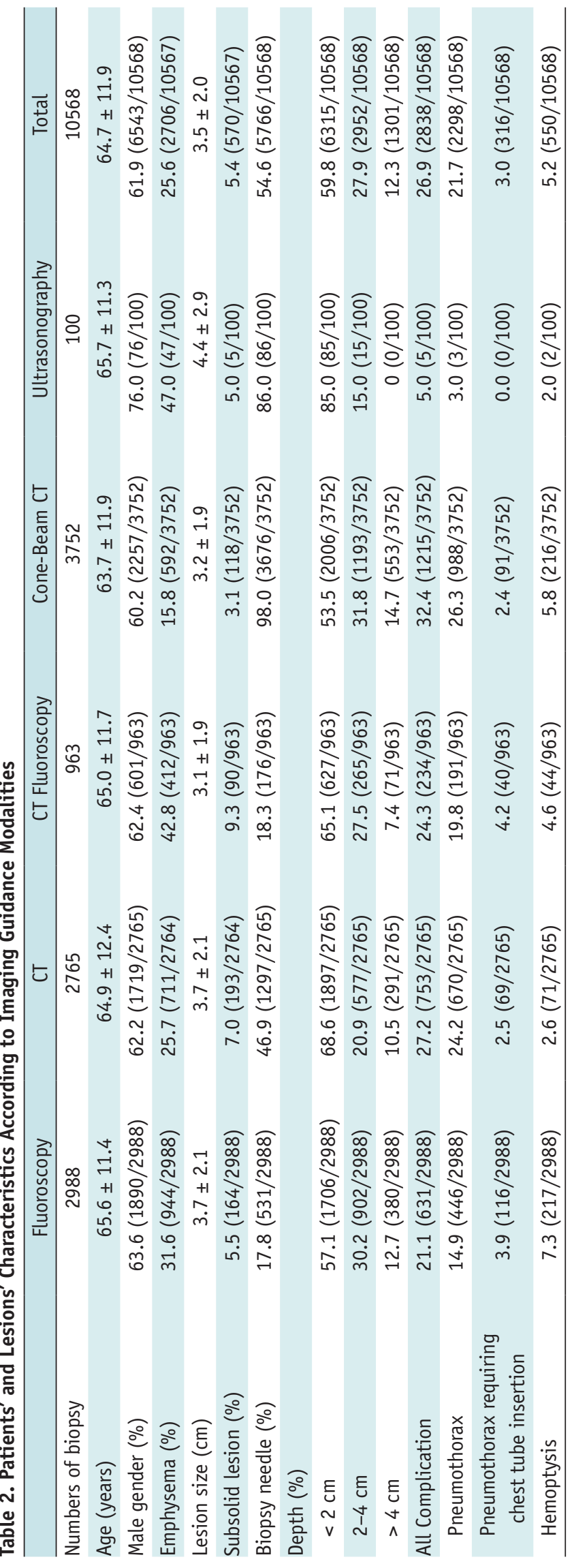


$0.001)$, and number of pleural passages ( $p<0.001-0.608)$.

In the multivariate logistic regression analysis including these factors (Table 3), pneumothorax was positively associated with all 3 CT-based guidance modalities. CT (odds ratio [OR], 2.752 [95\% CI, 2.325-3.258]; $p<0.001)$, CT fluoroscopy (OR, 1.440 [95\% CI, 1.176-1.762]; $p<0.001)$, and cone-beam CT (OR, 2.906; [95\% CI, 2.235-3.779]; $p<0.001$ ) were significant independent risk factors for pneumothorax, while ultrasonography was a significant independent protector for pneumothorax (OR, 0.170; [95\% CI, 0.053-0.547]; $p=0.003$ ).

Other independent risk factors included older age ( $O R$, 1.284; [95\% CI, 1.145-1.440]; $p<0.001)$, male sex (OR, 1.178; [95\% CI, 1.017-1.366]; $p=0.029)$, a lesion in the right middle lobe (OR, 1.446; [95\% CI, 1.192-1.754]; $p$ $<0.001)$, a lesion smaller than $2 \mathrm{~cm}(0 \mathrm{R}, 1.604$ [95\% CI, $1.442-1.784] ; p<0.001)$, subsolid composition (OR, 1.411

Table 3. Multivariate Logistic Regression Analysis of Risk Factors for Pneumothorax

\begin{tabular}{|c|c|c|}
\hline & $P$ & OR $(95 \% \mathrm{CI})$ \\
\hline Age $\geq 60$ years (vs. $<60$ years) & $<0.001^{*}$ & $1.284(1.145-1.440)$ \\
\hline Male (vs. female) & $0.029^{*}$ & $1.178(1.017-1.366)$ \\
\hline Smoking (vs. non-smoker) & 0.050 & $1.155(1.000-1.333)$ \\
\hline \multicolumn{3}{|l|}{ Guiding modality (vs. fluoroscopy) } \\
\hline CT & $<0.001^{*}$ & $2.752(2.325-3.258)$ \\
\hline CT fluoroscopy & $<0.001^{*}$ & $1.440(1.176-1.762)$ \\
\hline Cone-beam CT & $<0.001^{*}$ & $2.906(2.235-3.779)$ \\
\hline Ultrasonography & $0.003^{*}$ & $0.170(0.053-0.547)$ \\
\hline \multicolumn{3}{|l|}{ Lobe (vs. right upper lobe) } \\
\hline Right middle lobe & $<0.001^{*}$ & $1.446(1.192-1.754)$ \\
\hline Right lower lobe & 0.561 & $1.041(0.908-1.194)$ \\
\hline Left upper lobe & 0.992 & $1.001(0.875-1.145)$ \\
\hline Left lower lobe & 0.917 & $1.008(0.867-1.172)$ \\
\hline $\begin{array}{l}\text { Nodule of } 2 \mathrm{~cm} \text { or less } \\
\text { (vs. }>2 \mathrm{~cm})\end{array}$ & $<0.001^{*}$ & $1.604(1.442-1.784)$ \\
\hline Subsolid composition (vs. solid) & $0.001^{*}$ & $1.411(1.151-1.730)$ \\
\hline Emphysema & $<0.001^{*}$ & $1.548(1.372-1.747)$ \\
\hline \multicolumn{3}{|l|}{ Distance from pleura (vs. $\leq 2 \mathrm{~cm}$ ) } \\
\hline $2-4 \mathrm{~cm}$ & $<0.001^{*}$ & $1.805(1.617-2.015)$ \\
\hline$>4 \mathrm{~cm}$ & $<0.001^{*}$ & $2.518(2.189-2.896)$ \\
\hline Core biopsy (vs. FNA) & 0.660 & $1.072(0.786-1.463)$ \\
\hline \multicolumn{3}{|l|}{ Needle gauze (vs. 22 G) } \\
\hline $19-20 \mathrm{G}$ & 0.648 & $1.085(0.763-1.544)$ \\
\hline $17-18 \mathrm{G}$ & 0.549 & $0.895(0.623-1.286)$ \\
\hline \multicolumn{3}{|l|}{ Number of pleural passage (vs. 1) } \\
\hline 2 & $<0.001^{*}$ & $1.784(1.498-2.126)$ \\
\hline$\geq 3$ & $<0.001^{*}$ & $3.153(2.502-3.974)$ \\
\hline
\end{tabular}

*Statistical significance below $0.05 . \mathrm{CI}=$ confidence interval, $\mathrm{FNA}=$ fine-needle aspiration, $\mathrm{OR}=$ odds ratio
[95\% CI, 1.151-1.730]; $p=0.001)$, emphysema (OR, 1.548 [95\% CI, 1.372-1.747]; $p<0.001)$, a deep-seated lesion located between 2 and $4 \mathrm{~cm}$ from the pleura (OR, 1.805 [95\% CI, 1.617-2.015]; $p<0.001)$ or deeper than $4 \mathrm{~cm}(0 \mathrm{R}, 2.518$ [95\% CI, 2.189-2.896]; $p<0.001), 2$ pleural passages (OR, 1.784 [95\% CI, 1.498-2.126]; $p<0.001)$, and three or more pleural passages (0R, 3.153 [95\% CI, 2.502-3.974]; $p$ $<0.001)$. Smoking history was a marginally significant risk factor for pneumothorax (OR, 1.155 [95\% CI, 1.000-1.333]; $p=0.050)$.

\section{Pneumothorax Requiring Chest Tube Insertion}

In the univariate logistic regression analysis for pneumothorax requiring chest tube insertion (Supplementary Table 2 in the online-only Data Supplement), at least marginal statistical significance was found for older age $(p<0.001)$, male sex $(p<0.001)$, smoking history $(p$ $<0.001)$, guidance modality $(p<0.001-0.996)$, lobar location $(p=0.007-0.504)$, lesion diameter $(p=0.021)$, lesion composition $(p=0.046)$, emphysema $(p<0.001)$, a deep-seated lesion $(p<0.001)$, an immediate position change after PTNB $(p<0.001)$, biopsy method $(p<0.001)$, needle gauge ( $p=0.001-0.074)$, and the number of pleural passages $(p=0.001-0.047)$.

The multivariate logistic regression analysis revealed that none of the 3 CT-based guidance modalities, including CT (OR, 1.149 [95\% CI, 0.769-1.718]; $p=0.497)$, CT fluoroscopy (OR, $0.767[95 \% \mathrm{CI}, 0.501-1.174] ; p=0.222)$, and cone-beam CT (OR, 1.482 [95\% CI, 0.765-2.764]; $p=0.216)$ increased the risk of pneumothorax requiring chest tube insertion (Table 4). Older age (OR, 1.521 [95\% CI, 1.125-2.057]; $p=0.006$ ), a lesion smaller than $2 \mathrm{~cm}$ (OR, 1.383 [95\% CI, 1.075-1.780]; $p=0.012)$, subsolid composition (OR, 1.557 [95\% CI, 1.005-2.413]; $p=0.048)$, emphysema (OR, 2.745 [95\% CI, 2.104-3.581]; $p<0.001)$, a deep-seated lesion located between 2 and $4 \mathrm{~cm}$ from the pleura (OR, 2.779 [95\% CI, 2.117-3.647]; $p<0.001)$ or deeper than $4 \mathrm{~cm}(0 \mathrm{R}, 4.318$ [95\% CI, 3.167-5.886]; $p$ $<0.001$ ), and three or more pleural passages (OR, 2.024 [95\% CI, 1.282-3.195]; $p=0.002$ ) were other significant independent risk factors. A rapid needle-out patient-rollover approach was the sole significant independent protector $(0 R$, 0.600 [95\% CI, 0.439-0.820]; $p=0.001$ )

\section{Hemoptysis}

In the univariate analysis, female sex $(p<0.001)$, smoking history $(p<0.001)$, guidance modality $(p<0.001-$ 
Table 4. Multivariate Logistic Regression Analysis of Risk Factors for Pneumothorax Requiring Chest Tube Insertion

\begin{tabular}{|c|c|c|}
\hline & $P$ & OR $(95 \% \mathrm{CI})$ \\
\hline Age $\geq 60$ (vs. < 60) & $0.006^{*}$ & $1.521(1.125-2.057)$ \\
\hline Male (vs. female) & 0.693 & $1.081(0.734-1.592)$ \\
\hline Smoking (vs. non-smoker) & 0.137 & $1.326(0.914-1.923)$ \\
\hline \multicolumn{3}{|l|}{ Guiding modality (vs. fluoroscopy) } \\
\hline CT & 0.497 & $1.149(0.769-1.718)$ \\
\hline CT fluoroscopy & 0.222 & $0.767(0.501-1.174)$ \\
\hline Cone-beam CT & 0.216 & $1.482(0.765-2.764)$ \\
\hline Ultrasonography & 0.996 & Not available \\
\hline \multicolumn{3}{|l|}{ Lobe (vs. right upper lobe) } \\
\hline Right middle lobe & 0.499 & $0.849(0.528-1.365)$ \\
\hline Right lower lobe & 0.402 & $0.870(0.629-1.204)$ \\
\hline Left upper lobe & 0.114 & $0.783(0.578-1.060)$ \\
\hline Left lower lobe & 0.334 & $0.833(0.575-1.207)$ \\
\hline Nodule of $2 \mathrm{~cm}$ or less (vs. $>2 \mathrm{~cm}$ ) & $0.012^{*}$ & $1.383(1.075-1.780)$ \\
\hline Subsolid composition (vs. solid) & $0.048^{*}$ & $1.557(1.005-2.413)$ \\
\hline Emphysema & $<0.001^{*}$ & $2.745(2.104-3.581)$ \\
\hline \multicolumn{3}{|l|}{ Distance from pleura (vs. $\leq 2 \mathrm{~cm}$ ) } \\
\hline $2-4 \mathrm{~cm}$ & $<0.001^{*}$ & $2.779(2.117-3.647)$ \\
\hline$>4 \mathrm{~cm}$ & $<0.001^{*}$ & $4.318(3.167-5.886)$ \\
\hline $\begin{array}{l}\text { Rapid needle-out patient-rollover } \\
\text { approach }\end{array}$ & $0.001^{*}$ & $0.600(0.439-0.820)$ \\
\hline Core biopsy (vs. FNA) & 0.186 & $0.612(0.295-1.268)$ \\
\hline \multicolumn{3}{|l|}{ Needle gauze (vs. $22 \mathrm{G}$ ) } \\
\hline $19-20 \mathrm{G}$ & 0.640 & $1.230(0.517-2.925)$ \\
\hline $17-18 \mathrm{G}$ & 0.934 & $0.965(0.411-2.264)$ \\
\hline \multicolumn{3}{|l|}{ Number of pleural passage (vs. 1) } \\
\hline 2 & 0.178 & $1.286(0.892-1.853)$ \\
\hline$\geq 3$ & $0.002^{*}$ & $2.024(1.282-3.195)$ \\
\hline
\end{tabular}

*Statistical significance below 0.05 .

$0.061)$, lesion diameter $(p=0.001)$, lesion composition ( $p$ $<0.001)$, emphysema $(p<0.001)$, a deep-seated lesion ( $p$ $<0.001)$, needle gauge $(p=0.003-0.182)$, and the number of pleural passages ( $p=0.011-0.499)$ showed statistical significance (Supplementary Table 3 in the online-only Data Supplement). The multivariate analysis included those variables, and we added the biopsy method ( $p=0.295)$ to the final model.

In comparison with fluoroscopy, all 3 CT-based guidance modalities, namely, CT (OR, 0.348 [95\% CI, 0.247-0.491]; $p$ $<0.001$ ), CT fluoroscopy (OR, 0.594 [95\% CI, 0.419-0.843]; $p=0.004)$, and cone-beam CT (OR, 0.479; [95\% CI, 0.317 $0.724] ; p<0.001)$ were significant independent protectors against hemoptysis (Table 5). In addition, female sex (OR, 1.495 [95\% CI, 1.154-1.937]; $p=0.002)$, a lesion smaller than $2 \mathrm{~cm}(0 \mathrm{R}, 1.226$ [95\% CI, 1.012-1.486]; $p=0.037)$, subsolid composition (OR, 1.685 [95\% CI, 1.232-2.304]; $p$
Table 5. Multivariate Logistic Regression Analysis of Risk Factors for Hemoptysis

\begin{tabular}{|c|c|c|}
\hline & $P$ & OR $(95 \% \mathrm{CI})$ \\
\hline Female (vs. male) & $0.002^{*}$ & $1.495(1.154-1.937)$ \\
\hline Smoking (vs. non-smoker) & 0.058 & $0.773(0.593-1.008)$ \\
\hline \multicolumn{3}{|l|}{ Guiding modality (vs. fluoroscopy) } \\
\hline CT & $<0.001^{*}$ & $0.348(0.247-0.491)$ \\
\hline CT fluoroscopy & 0.004 & $0.594(0.419-0.843)$ \\
\hline Cone-beam CT & $<0.001^{*}$ & $0.479(0.317-0.724)$ \\
\hline Ultrasonography & 0.093 & $0.294(0.070-1.228)$ \\
\hline Nodule of $2 \mathrm{~cm}$ or less (vs. $>2 \mathrm{~cm}$ ) & $0.037^{*}$ & $1.226(1.012-1.486)$ \\
\hline Subsolid composition (vs. solid) & $0.001^{*}$ & $1.685(1.232-2.304)$ \\
\hline Emphysema & $0.024^{*}$ & $0.740(0.570-0.961)$ \\
\hline \multicolumn{3}{|l|}{ Distance from pleura (vs. $\leq 2 \mathrm{~cm}$ ) } \\
\hline $2-4 \mathrm{~cm}$ & $<0.001^{*}$ & $1.573(1.288-1.922)$ \\
\hline$>4 \mathrm{~cm}$ & $<0.001^{*}$ & $2.531(2.003-3.199)$ \\
\hline Core biopsy (vs. FNA) & $0.049^{*}$ & $1.595(1.001-2.541)$ \\
\hline \multicolumn{3}{|l|}{ Needle gauze (vs. 22 G) } \\
\hline $19-20 \mathrm{G}$ & 0.164 & $0.653(0.359-1.189)$ \\
\hline $17-18 \mathrm{G}$ & 0.945 & $0.981(0.565-1.701)$ \\
\hline \multicolumn{3}{|l|}{ Number of pleural passage (vs. 1) } \\
\hline 2 & 0.490 & $1.107(0.829-1.479)$ \\
\hline$\geq 3$ & 0.219 & $0.760(0.491-1.177)$ \\
\hline
\end{tabular}

*Statistical significance below 0.05 .

$=0.001)$, and a deep-seated lesion located between 2 and $4 \mathrm{~cm}$ from the pleura (OR, 1.573 [95\% CI, 1.288-1.922]; $p$ $<0.001)$ or deeper than $4 \mathrm{~cm}(0 \mathrm{R}, 2.531$ [95\% CI, 2.0033.199]; $p<0.001)$, and core biopsy (OR, 1.595 [95\% CI, $1.001-2.541] ; p=0.049)$ were significant risk factors for hemoptysis, while emphysema (0R, 0.740 [95\% CI, 0.570$0.961] ; p=0.024$ ) was a significant protective factor.

\section{DISCUSSION}

This large retrospective cohort study revealed that hemoptysis occurred less frequently when using CTbased guidance modalities. Although pneumothorax requiring chest tube insertion showed a similar incidence, pneumothorax was more frequently detected using CT-based guidance modalities.

In comparison with fluoroscopy, CT (OR, 0.348 [95\% CI, 0.247-0.491]; $p<0.001)$, CT fluoroscopy (OR, 0.594 [95\% CI, 0.419-0.843]; $p=0.004)$, and cone-beam CT (OR, 0.479; [95\% CI, 0.317-0.724]; $p<0.001$ ) were significant independent protectors against hemoptysis. CT and CTbased guidance modalities are more advantageous than fluoroscopy for preprocedural planning and intraprocedural tracking of needle placement. Furthermore, penetration 
and cutting injuries of the large vessels and bronchi can be avoided by reviewing the location of the needle tip before aspiration or firing the biopsy gun (4). This precise anatomic evaluation could potentially reduce the incidence of hemoptysis. In line with the results of a prior publication (10), needle gauge and the number of pleural penetrations were not associated with hemoptysis.

In the multivariate analysis, female sex, lesions smaller than $2 \mathrm{~cm}$, subsolid composition, deep-seated lesions, and core biopsy also showed statistical significance, and these are well-recognized risk factors for hemoptysis, as hemoptysis is associated with the less compact nature of small subsolid lesions compared to large solid lesions and the higher chances of tissue injuries in core biopsy than in FNA (10-13). The higher prevalence of subsolid lesions in female patients might be partly responsible for the higher incidence of hemoptysis in female patients, although both lesion composition and sex were adjusted in the multivariate analysis. Interestingly, emphysema was found to be a significant protector against hemoptysis. Smoking induces pulmonary vascular remodeling, and in patients with severe emphysema, pulmonary capillaries decrease due to apoptosis of the pulmonary epithelium (14). These pathophysiologic changes may be associated with the lower incidence of hemoptysis after PTNB. A larger prospective study will help clarify the association of emphysema with hemoptysis.

The higher incidence of pneumothorax in PTNBs performed using CT-based guidance modalities than in PTNBs performed using fluoroscopy is presumed to be a detection issue rather than a true increase in the occurrence of pneumothorax. In the CT-based guidance modalities, the presence of pneumothorax was usually evaluated on CT images that were taken during the procedure and immediately after the completion of PTNB; thus, even a minute amount of pneumothorax could be found. In contrast, post-procedural chest radiography was used for assessing the presence of pneumothorax in fluoroscopyguided PTNB. On chest radiographs, even a considerable amount of pneumothorax can be missed (15). The finding showing similar incidence rates of pneumothorax requiring tube insertion across guidance modalities supports the proposal that the more sensitive detection of pneumothorax resulted in the higher overall incidence of pneumothorax in the CT-based guidance modalities.

The independent risk factors for pneumothorax included older age, male sex, a history of smoking, a lesion in the right middle lobe, a lesion smaller than $2 \mathrm{~cm}$, a subsolid lesion, emphysema, a deep-seated lesion, and multiple pleural passages. Indeed, relevant studies have indicated that smaller lesions and deeper parenchymal and greater number of pleural penetrations during the PTNB result in more frequent pneumothorax, and most of the aforementioned risk factors are in agreement with prior studies $(11,16-18)$, except for a lesion in the right middle lobe and subsolid composition of the lesion. The higher incidence of pneumothorax in older patients and male patients potentially results from the reduced lung elasticity with aging (19) and the higher recoil pressure in male patients (20). As the right middle lobe is surrounded by major and minor fissures, a transfissural approach may be more frequently required for a right middle lobar lesion than for other lobar lesions (17). We were not able to determine whether a PTNB was performed via a transfissural route, and the higher incidence of pneumothorax in right middle lobar lesions may be associated with the more frequent use of transfissural approaches. A higher incidence of pneumothorax in subsolid lesions may be explainable by the weaker compression of internal bronchial structures by loose pathologic tissue after the biopsy injury than is the case for solid lesions (21). Further investigation is warranted to confirm the association between pneumothorax and subsolid lesions.

The sole independent protector against pneumothorax requiring chest tube insertion was a rapid needle-out patient-rollover approach. The patient biopsy-side-down position potentially reduces the incidence of pneumothorax by decreasing the size of the alveoli surrounding the needle track, subsequently causing airway closure and elevated resistance to collateral ventilation in the dependent lung $(22,23)$. We validated the observations recorded by 0 'Neill et al. (22) in a large population. Nevertheless, some discrepancies exist between their results and ours: this approach reduced the overall pneumothorax rate in the study of $0^{\prime}$ Neill et al. (22), but not in ours. Vigorous turnover was not uniformly applicable to all patients in our clinical practice, and uncontrolled respiration by the patient during a position change may cause a small amount of air leakage through the puncture site or needle tract, as observed by Kim et al. (23). The independent risk factors for pneumothorax requiring chest tube insertion were a lesion smaller than $2 \mathrm{~cm}$, a subsolid lesion, emphysema, a deep-seated lesion, and multiple pleural passages (three times or higher); these risk factors are consistent with prior 
observations $(4,17,18)$.

Interestingly, a lesion in the right middle lobe and twice-pleural passages were risk factors for pneumothorax but not for pneumothorax requiring chest tube insertion. These discrepancies may be partly explained by the amount of pneumothorax after biopsy: the large amount of pneumothorax requiring tube drainage is less likely to occur with twice-pleural passages or a potential transfissural approach. In addition, neither needle gauze nor core biopsy were risk factors for pneumothorax or pneumothorax requiring chest tube insertion. This observation indicates that the size of the pleural injury when the needle penetrates a pleura did not affect the overall occurrence of pneumothorax but potentially resulted in non-immediate pneumothorax (18).

Hemothorax is one of the rare complications after PTNB. It occurred in $0.57 \%$ of the biopsies, which was consistent with prior observations $(0.20-0.92 \%)(11,24$, 25). There were two potential risk factors for hemothorax: prone position and core biopsy. The association between hemothorax and prone position may result from the varying vertical position of intercostal vessels within the intercostal space around the spine where the intercostal vessel is not shielded by the intercostal groove of the superior rib (26). Similarly, in core biopsy, intercostal vessels may be exposed to the active part of the biopsy needle and may be subsequently injured when PTNB is performed for a subpleural lesion (27). Another rare complication after PTNB is air embolism. The incidence of air embolism in our population was $0.02 \%$, which was consistent with the reported incidence (range, $0.02-0.07 \%$ ) in relevant publications $(25,28,29)$.

This study has several limitations, as our cohort was collected in a retrospective manner. The impact of guiding modalities on complication rates in PTNB was evaluated without considering a hospital effect. As mentioned earlier, the choice of guidance modality and usage of a coaxial needle showed an extremely uneven distribution among the participating institutions. Thoracic radiologists have their own preferences for a particular guidance modality and for the usage of a coaxial or non-coaxial needle; such variations could not be resolved in an observational cohort study, and we were not able to appropriately evaluate the hospital effect on complications of PTNB. Nevertheless, given the similar referral settings and experience levels of the affiliated thoracic radiologists across the participating hospitals, we believe that our approach could evaluate the impact of CT-based imaging modalities for needle guidance on complications in the study population. The use of a transfissural approach for PTNB was not analyzed in our population. This confounder may have affected the results of our risk factor analysis for pneumothorax. In addition, although we regarded repeat biopsies for the same lesion as separate initial PTNBs, pulmonary lesions demanding a repeated biopsy may have specific characteristics affecting the complication rate.

In conclusion, when compared with fluoroscopy, hemoptysis occurred less frequently using CT-based guidance modalities; while pneumothorax requiring chest tube insertion showed a similar incidence, pneumothorax was more frequently detected. A rapid needle-out patientrollover approach was effective for reducing the need for chest tube insertion due to pneumothorax. Understanding the benefits and limitations of CT-based guidance modalities in terms of complications will be helpful for selecting the appropriate guidance modality for PTNB.

\section{Supplementary Materials}

The online-only Data Supplement is available with this article at https://doi.org/10.3348/kjr.2018.0064.

\section{Conflicts of Interest}

The authors have no financial conflicts of interest.

ORCID

Chang Min Park

https://orcid.org/0000-0003-1884-3738

Soon Ho Yoon

https://orcid.org/0000-0002-3700-0165

\section{REFERENCES}

1. Manhire A, Charig M, Clelland C, Gleeson F, Miller R, Moss H, et al.; BTS. Guidelines for radiologically guided lung biopsy. Thorax 2003;58:920-936

2. Rivera MP, Mehta AC, Wahidi MM. Establishing the diagnosis of lung cancer: diagnosis and management of lung cancer, 3rd ed: American College of Chest Physicians evidence-based clinical practice guidelines. Chest 2013;143(5 Suppl):e142S-e165S

3. Hiraki T, Mimura H, Gobara H, Iguchi T, Fujiwara H, Sakurai $\mathrm{J}$, et al. CT fluoroscopy-guided biopsy of 1,000 pulmonary lesions performed with 20-gauge coaxial cutting needles: diagnostic yield and risk factors for diagnostic failure. Chest 2009;136:1612-1617 
4. Lee SM, Park CM, Lee KH, Bahn YE, Kim JI, Goo JM. C-arm cone-beam CT-guided percutaneous transthoracic needle biopsy of lung nodules: clinical experience in 1108 patients. Radiology 2014;271:291-300

5. Wu CC, Maher MM, Shepard JA. Complications of CT-guided percutaneous needle biopsy of the chest: prevention and management. AJR Am J Roentgenol 2011;196:W678-W682

6. Heerink WJ, de Bock GH, de Jonge GJ, Groen HJ, Vliegenthart R, Oudkerk M. Complication rates of CT-guided transthoracic lung biopsy: meta-analysis. Eur Radiol 2017;27:138-148

7. Kim GR, Hur J, Lee SM, Lee HJ, Hong YJ, Nam JE, et al. CT fluoroscopy-guided lung biopsy versus conventional CT-guided lung biopsy: a prospective controlled study to assess radiation doses and diagnostic performance. Eur Radiol 2011;21:232239

8. Prosch H, Stadler A, Schilling M, Bürklin S, Eisenhuber E, Schober $E$, et al. CT fluoroscopy-guided vs. multislice CT biopsy mode-guided lung biopsies: accuracy, complications and radiation dose. Eur J Radiol 2012;81:1029-1033

9. Rotolo N, Floridi C, Imperatori A, Fontana F, Ierardi AM, Mangini $M$, et al. Comparison of cone-beam CT-guided and CT fluoroscopy-guided transthoracic needle biopsy of lung nodules. Eur Radiol 2016;26:381-389

10. Tai R, Dunne RM, Trotman-Dickenson B, Jacobson FL, Madan $R$, Kumamaru KK, et al. Frequency and severity of pulmonary hemorrhage in patients undergoing percutaneous CT-guided transthoracic lung biopsy: single-institution experience of 1175 cases. Radiology 2016;279:287-296

11. Yeow KM, Su IH, Pan KT, Tsay PK, Lui KW, Cheung YC, et al. Risk factors of pneumothorax and bleeding: multivariate analysis of 660 CT-guided coaxial cutting needle lung biopsies. Chest 2004;126:748-754

12. Hwang EJ, Park CM, Yoon SH, Lim HJ, Goo JM. Risk factors for haemoptysis after percutaneous transthoracic needle biopsies in 4,172 cases: focusing on the effects of enlarged main pulmonary artery diameter. Eur Radiol 2018;28:1410-1419

13. Lee SM, Park CM, Song YS, Kim H, Kim YT, Park YS, et al. CT assessment-based direct surgical resection of part-solid nodules with solid component larger than $5 \mathrm{~mm}$ without preoperative biopsy: experience at a single tertiary hospital. Eur Radiol 2017;27:5119-5126

14. Siafakas NM, Antoniou KM, Tzortzaki EG. Role of angiogenesis and vascular remodeling in chronic obstructive pulmonary disease. Int J Chron Obstruct Pulmon Dis 2007;2:453-462

15. Omar HR, Abdelmalak H, Mangar D, Rashad R, Helal E, Camporesi EM. Occult pneumothorax, revisited. J Trauma Manag Outcomes 2010;4:12

16. Geraghty PR, Kee ST, McFarlane G, Razavi MK, Sze DY, Dake MD. CT-guided transthoracic needle aspiration biopsy of pulmonary nodules: needle size and pneumothorax rate. Radiology 2003;229:475-481

17. Boskovic T, Stanic J, Pena-Karan S, Zarogoulidis P, Drevelegas K, Katsikogiannis N, et al. Pneumothorax after transthoracic needle biopsy of lung lesions under CT guidance. J Thorac Dis 2014;6 Suppl 1:S99-S107

18. Lim WH, Park CM, Yoon SH, Lim HJ, Hwang EJ, Lee JH, et al. Time-dependent analysis of incidence, risk factors and clinical significance of pneumothorax after percutaneous lung biopsy. Eur Radiol 2018;28:1328-1337

19. Sharma G, Goodwin J. Effect of aging on respiratory system physiology and immunology. Clin Interv Aging 2006;1:253-260

20. Colebatch HJ, Greaves IA, Ng CK. Exponential analysis of elastic recoil and aging in healthy males and females. J Appl Physiol Respir Environ Exerc Physiol 1979;47:683-691

21. Yamagami T, Yoshimatsu R, Miura H, Yamada K, Takahata A, Matsumoto $T$, et al. Diagnostic performance of percutaneous lung biopsy using automated biopsy needles under CTfluoroscopic guidance for ground-glass opacity lesions. $\mathrm{Br} \mathrm{J}$ Radiol 2013;86:20120447

22. O'Neill AC, McCarthy C, Ridge CA, Mitchell $P$, Hanrahan E, Butler $M$, et al. Rapid needle-out patient-rollover time after percutaneous CT-guided transthoracic biopsy of lung nodules: effect on pneumothorax rate. Radiology 2012;262:314-319

23. Kim JI, Park CM, Lee SM, Goo JM. Rapid needle-out patientrollover approach after cone beam CT-guided lung biopsy: effect on pneumothorax rate in 1,191 consecutive patients. Eur Radiol 2015;25:1845-1853

24. Weinberg B, Watumull L, Landay M, Omar H. Variable presentations of thoracic biopsy related hemothorax. J Clin Med 2013;1:22-27

25. Tomiyama N, Yasuhara Y, Nakajima Y, Adachi S, Arai Y, Kusumoto $M$, et al. CT-guided needle biopsy of lung lesions: a survey of severe complication based on 9783 biopsies in Japan. Eur J Radiol 2006;59:60-64

26. Helm EJ, Rahman NM, Talakoub 0, Fox DL, Gleeson FV. Course and variation of the intercostal artery by CT scan. Chest 2013;143:634-639

27. Yoshimatsu R, Yamagami T, Tanaka 0, Miura H, Tanaka T, Suzuki T, et al. Comparison of fully automated and semiautomated biopsy needles for lung biopsy under CT uoroscopic guidance. Br J Radiol 2012;85:208-213

28. Richardson CM, Pointon KS, Manhire AR, Macfarlane JT. Percutaneous lung biopsies: a survey of UK practice based on 5444 biopsies. Br J Radiol 2002;75:731-735

29. Hiraki T, Fujiwara H, Sakurai J, Iguchi T, Gobara H, Tajiri N, et al. Nonfatal systemic air embolism complicating percutaneous CT-guided transthoracic needle biopsy: four cases from a single institution. Chest 2007;132:684-690 